\title{
Inhibition of protein SUMOylation by natural quinones
}

\author{
Isao Fukuda ${ }^{1,7}$, Mikako Hirohama ${ }^{1,7}$, Akihiro Ito ${ }^{1,2}$, Mohammad Tariq ${ }^{1,3}$, Yasuhiro Igarashi ${ }^{4}$, Hisato Saitoh ${ }^{5}$ \\ and Minoru Yoshida ${ }^{1,2,3,6}$
}

The Journal of Antibiotics (2016) 69, 776-779; doi:10.1038/ja.2016.23; published online 9 March 2016

Conjugation of small ubiquitin-related modifier (SUMO) to target proteins (SUMOylation) is a posttranslational modification that regulates a wide range of biological processes, including transcription, intracellular transport, DNA repair, DNA replication and cell signaling. ${ }^{1}$ SUMOylation is catalyzed by a multi-step enzymatic cascade that resembles ubiquitination: the process involves an E1-activating enzyme (a heterodimer consisting of Aos1 and Uba2), an E2-conjugating enzyme (Ubc9) and various E3 ligases. ${ }^{1}$ E3s catalyze SUMO conjugation to target proteins and may contribute to substrate specificity. SUMO is subsequently cleaved from target proteins by SUMO-specific isopeptidases. Because SUMO modifications have been implicated in a variety of disorders such as cancers ${ }^{2}$ and neurodegenerative diseases, ${ }^{3}$ the enzymes involved in SUMOylation are promising targets for therapeutic agents.

Several small-molecule inhibitors of SUMOylation have been identified. The first class of SUMOylation inhibitors to be characterized were ginkgolic acid and anacardic acid, which are isolated from the extract of Ginkgo biloba leaves. ${ }^{4}$ Subsequently, natural products, including two antibiotics (kerriamycin $\mathrm{B}^{5}$ and spectomycin $\mathrm{B}^{6}$ ) and davidiin from the plant Davidia involucrata, ${ }^{7}$ as well as synthetic compounds, ${ }^{8-11}$ have been shown to inhibit protein SUMOylation. In this study, we identified another class of natural products, which includes nocardione $\mathrm{A}$ and $\beta$-lapachone, that act as SUMOylation inhibitors. The results of mode-of-action studies suggested that these quinone-related compounds inhibit SUMOylation by oxidizing thiols of E1 and E2 enzymes through reactive oxygen species (ROS).

The materials for this study were obtained as follows. Goat polyclonal anti-SUMO-1 (N-19) and anti-UBC9 (N-15) antibodies were purchased from Santa Cruz Biotechnology (Dallas, TX, USA). Mouse monoclonal anti-T7 antibody was purchased from Merck Millipore (Billerica, MA, USA). Mouse monoclonal anti- $\alpha$-tubulin (B-5-1-2) and anti-FLAG (M2) antibodies were purchased from Sigma (St Louis, MO, USA). CM-H2DCFDA was purchased form Life Technologies (Carlsbad, CA, USA). Plasmids for expression of His-tagged SUMO-1, His- and T7-tagged RanGAP1-C2, GST-Aos1/
Uba2, and His-tagged Ubc9 plasmids and biotinylated SUMO-1 were described previously. ${ }^{4}$ 293T, HeLa, and A549 cells were maintained at $37^{\circ} \mathrm{C}$ in $5 \% \mathrm{CO}_{2}$ in Dulbecco's modified Eagle's medium supplemented with L-glutamine and $10 \%$ fetal bovine serum (GIBCO, Carlsbad, CA, USA). In vitro and in vivo SUMOylation assays were performed as described. ${ }^{4}$ The measurement of intracellular ROS was performed as follows. A549 cells suspended in phosphate-buffered saline were incubated with $10 \mu \mathrm{M} C \mathrm{CM}-\mathrm{H} 2 \mathrm{DCFDA}$ for $15 \mathrm{~min}$ at $37^{\circ} \mathrm{C}$, and then equal number of cells were distributed to the 96-well plate. After incubation for $30 \mathrm{~min}$ at $37^{\circ} \mathrm{C}$, the fluorescence intensity was measured at $\lambda_{\mathrm{ex}} 488 \mathrm{~nm} / \lambda_{\mathrm{em}} 520 \mathrm{~nm}$ with a SpectraMax M2 microplate reader (Molecular Devices, Sunnyvale, CA, USA). Assay for dithiothreitol (DTT)-sensitive crosslinking between E1 and E2 was performed as previously described. ${ }^{12}$

In situ cell-based screening ${ }^{4}$ of small-molecule libraries identified several compounds that inhibit SUMOylation, including four chemicals with ortho-quinone in their structures. These hit compounds included nocardione A, originally isolated as a tyrosine phosphatase inhibitor with antifungal activity, ${ }^{13}$ and its analogs 3,3-DINORdunnione, 3,3-DINOR-dehydrodunnione and $\beta$-lapachone, which were originally isolated from the bark of the lapacho tree (Figure 1a). ${ }^{14}$ In vitro SUMOylation assay confirmed that these four ortho-quinone-containing compounds inhibited in vitro protein SUMOylation in a dose-dependent manner (Figure 1b). The 50\% inhibitory concentration $\left(\mathrm{IC}_{50}\right)$ values of nocardione A, 3,3-DINORdunnione, 3,3-DINOR-dehydrodunnione and $\beta$-lapachone were 3.9, $1.1,1.2$ and $0.4 \mu \mathrm{M}$, respectively (Figure $1 \mathrm{c}$ ).

We next asked whether these quinone-containing compounds could inhibit in vivo protein SUMOylation by analyzing their effects in 293T cells expressing FLAG-tagged SUMO-1 (Figure 1d). Immunoblotting with an anti-FLAG antibody revealed that all compounds reduced the levels of high-MW SUMO conjugates at concentrations in the range $10-50 \mu \mathrm{M}$. The relatively weak in vivo inhibition of protein SUMOylation by nocardione A may reflect its relatively high in vitro $\mathrm{IC}_{50}$ and/or its relatively poor membrane permeability ascribable to a

${ }^{1}$ Chemical Genetics Laboratory, RIKEN, Saitama, Japan; ${ }^{2}$ Chemical Genomics Research Group, RIKEN Center for Sustainable Resource Science, Saitama, Japan; ${ }^{3}$ Graduate School of Science and Engineering, Saitama University, Saitama, Japan; ${ }^{4}$ Biotechnology Research Center, Toyama Prefectural University, Toyama, Japan; ${ }^{5}$ Department of New Frontier Sciences, Graduate School of Science and Technology, Kumamoto University, Kumamoto, Japan and ${ }^{6}$ Japan Agency for Medical Research and Development, AMED-CREST, Tokyo, Japan

${ }^{7}$ These authors contributed equally to this work.

Correspondence: Dr A Ito, Chemical Genetics Laboratory, RIKEN, 2-1 Hirosawa, Wako, Saitama 351-0198, Japan.

E-mail: akihiro-i@riken.jp

Received 27 January 2016; revised 2 February 2016; accepted 3 February 2016; published online 9 March 2016 
<smiles>CC1CC2=C(O1)c1cccc(O)c1C2=O</smiles>

Nocardione A<smiles>CC1CC2=C(O1)c1ccccc1C(=O)C2=O</smiles>

3,3-DINOR-dunnione<smiles>Cc1cc2c(o1)-c1ccccc1C(=O)C2=O</smiles>

3,3-DINOR-dehydrodunnione<smiles>CC1(C)CCC2=C(O1)c1ccccc1C(=O)C2=O</smiles>

$\beta$-Lapachone b

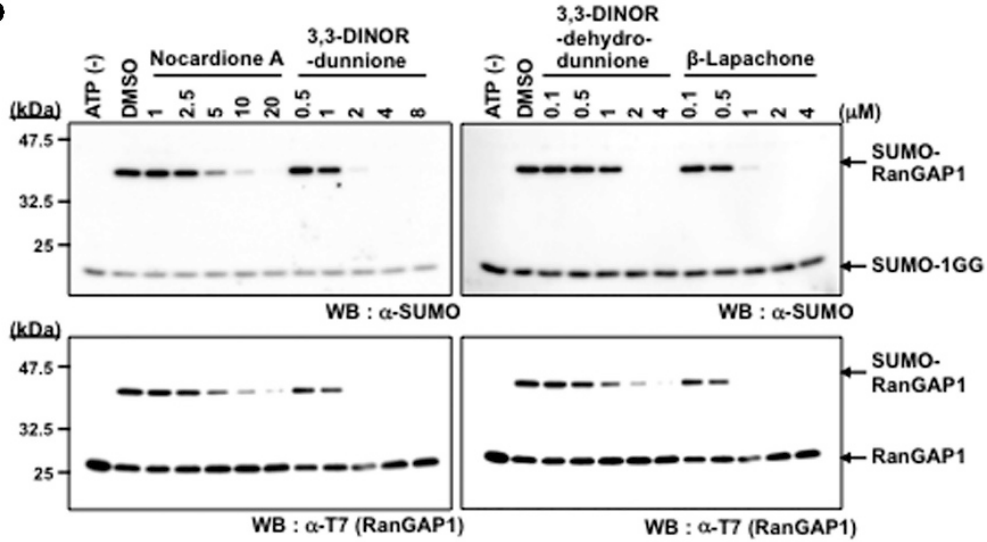

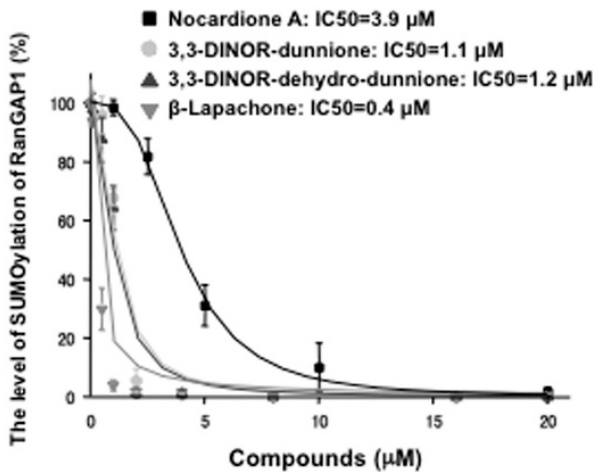
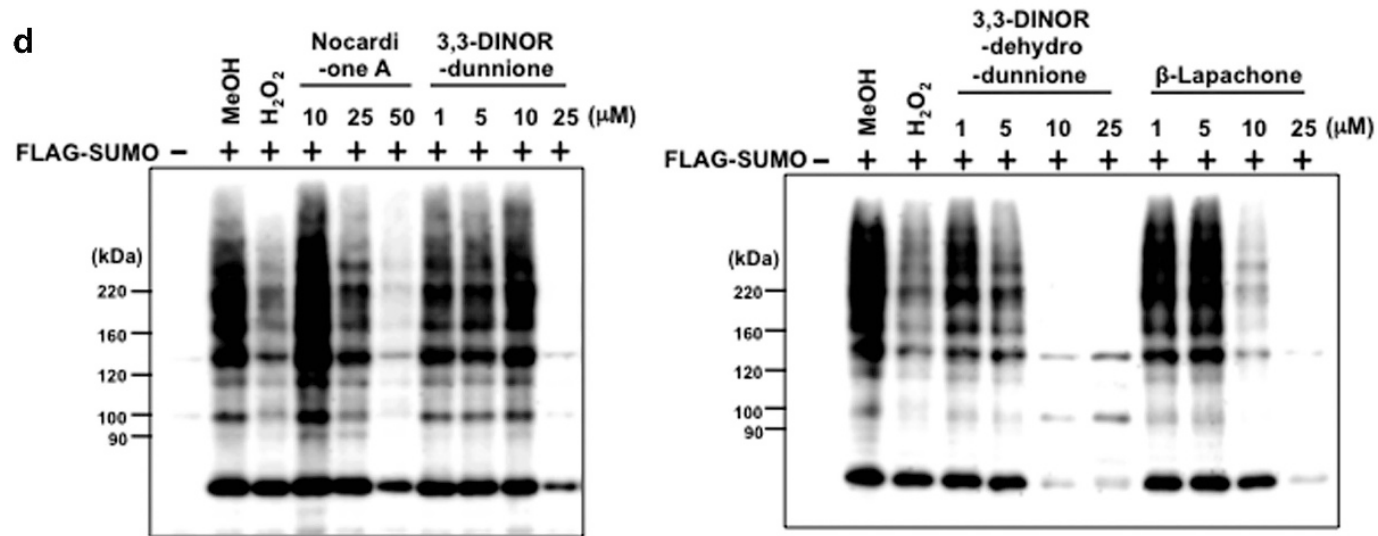

FLAG-SUMO- + $++t+t+t+$

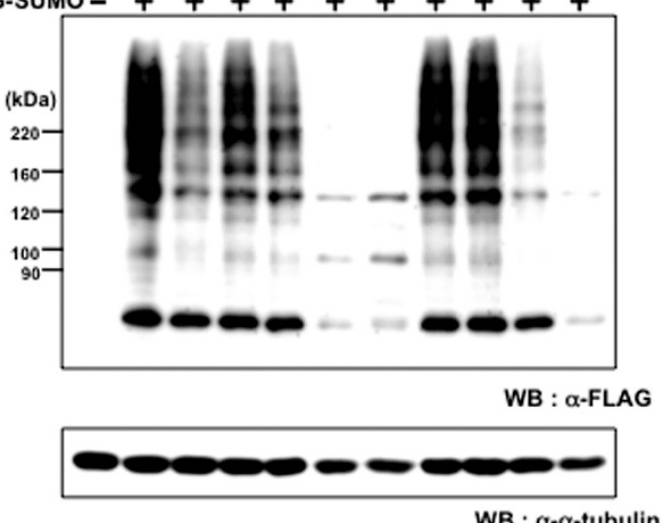

WB : $\alpha$ - $\alpha$-tubulin

WB : $\alpha-\alpha-$ tubulin

Figure 1 Quinone-related compounds inhibit protein SUMOylation. (a) Structure of nocardione A, 3,3-DINOR-dunnione, 3,3-DINOR-dehydrodunnione and $\beta$-lapachone. (b) Inhibition of in vitro SUMOylation by quinone-related compounds. Compounds at the indicated concentrations $(0.1-20 \mu \mathrm{m})$ were added to a reaction mixture containing His-tagged SUMO-1, His- and T7-tagged RanGAP1-C2, GST-Aos1/Uba2 (E1) and His-tagged-Ubc9 (E2) in the presence of ATP. SUMOylated RanGAP1-C2 was detected by immunoblotting using an anti-T7 or anti-SUMO-1 antibody. (c) IC 50 values of compounds for inhibition of SUMOylation of RanGAP1-C2 in vitro. The level of SUMOylation of RanGAP1-C2 was determined by measuring the intensity of the band corresponding to SUMOylated RanGAP1-C2, using Image Gauge version 4.22 (FUJIFILM, Tokyo, Japan). Error bars show the s.d.'s from three independent assays, and the $\mathrm{IC}_{50}$ value was calculated by comparison with a control sample incubated without the test compounds (the control SUMOylation level was defined as $100 \%$ ). (d) Inhibition of in vivo SUMOylation by quinone-related compounds. 293T cells were transfected with FLAG-tagged SUMO, and then treated with various concentrations of compounds $(1-50 \mu \mathrm{m})$ for $6 \mathrm{~h}$ or $1 \mathrm{~mm} \mathrm{H}_{2} \mathrm{O}_{2}$ for $1 \mathrm{~h}$. Cells were lysed in radioimmunoprecipitation assay buffer containing 50 mM $\mathrm{N}$ ethylmaleimide, and the lysates were separated by $6 \%$ SDS-polyacrylamide gel electrophoresis followed by immunoblotting with anti-FLAG antibody.

phenolic hydroxyl group in nocardione A. These results indicated that nocardione $\mathrm{A}$ and its derivatives 3,3-DINOR-dunnione, 3,3-DINORdehydrodunnione and $\beta$-lapachone are a novel class of SUMOylation inhibitors.

Because these natural products share ortho-quinone as a common skeleton in their structures (Figure 1a), ortho-quinone may be responsible for inhibition of SUMOylation. Quinones are highly redox-active molecules that generate ROS, including hydrogen peroxide, superoxide and hydroxyl radical. ${ }^{15}$ Indeed, nocardione A generated intracellular ROS in a dose-dependent manner (Figure 2a). Notably in this regard, ROS such as hydrogen peroxide have been shown to inhibit SUMO conjugation. ${ }^{12}$ Consistent with this previous observation, treatment with hydrogen peroxide reduced the level of high-MW SUMO conjugates in cells (Figure 1d). Accordingly, 
a

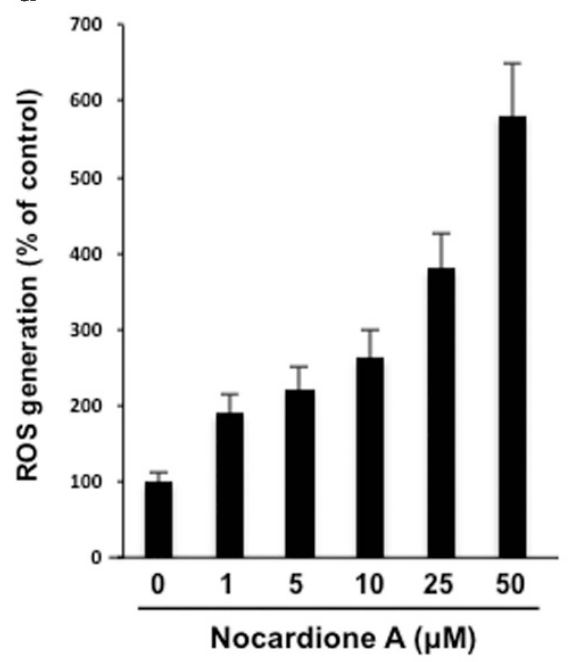

b

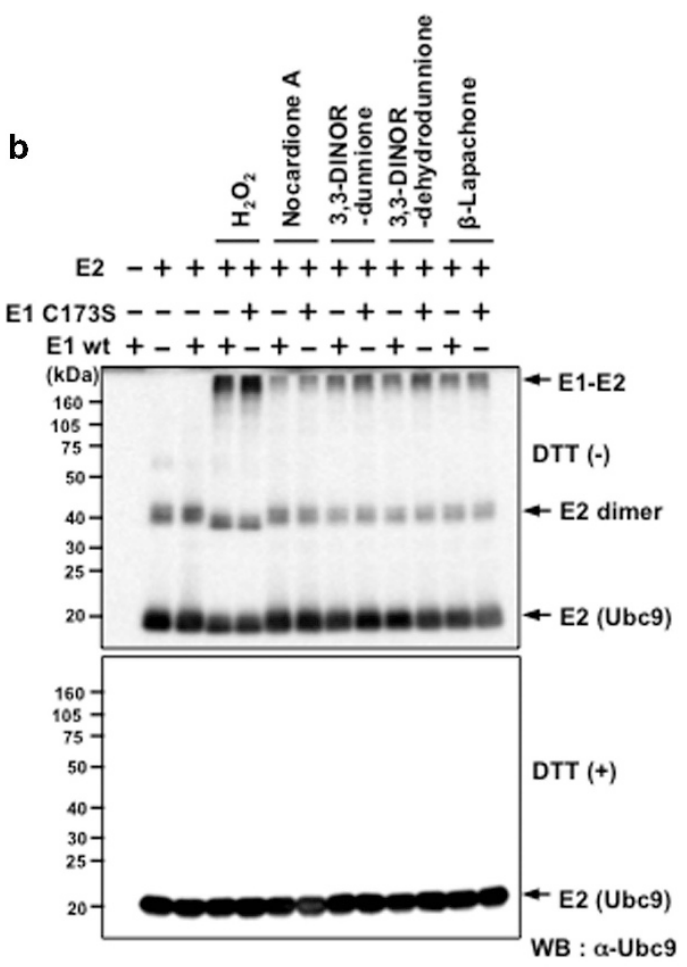

WB : $\alpha-U b c 9$
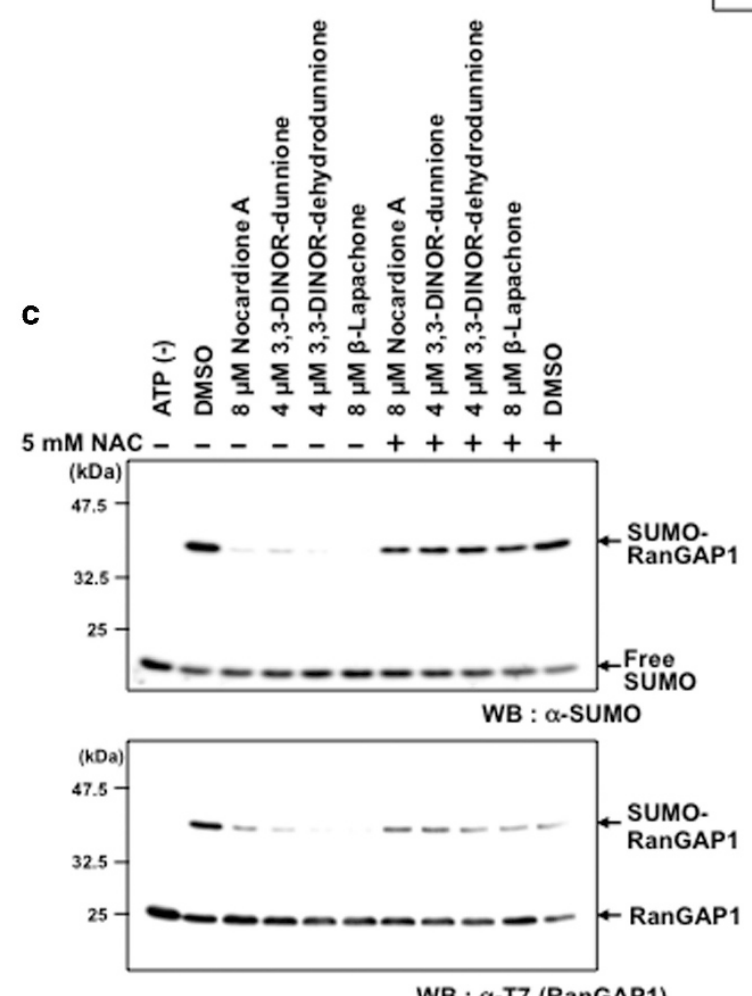

WB : $\alpha-$ T7 (RanGAP1)

Figure 2 Quinone-related compounds inhibit SUMOylation through reactive oxygen species (ROS) formation. (a) Generation of ROS by nocardione A. A549 cells were treated with nocardione $\mathrm{A}$ at the indicated concentrations for $30 \mathrm{~min}$ and the level of intracellular ROS was measured using CM-H2DCFDA. (b) Quinone-related compounds induce dithiothreitol (DTT)-sensitive crosslinking between E1 and E2 proteins. Either $1 \mathrm{~mm} \mathrm{H}_{2} \mathrm{O}_{2}$ or $10 \mu \mathrm{m}$ of the indicated compound was added to a reaction mixture containing $50 \mathrm{ng} \mathrm{\mu l}^{-1}$ of GST-Aos1/Uba2 or GST-Aos1/Uba2 C173S mutant, and $25 \mathrm{ng} \mu \mathrm{l}^{-1}$ of His-tagged-Ubc9 in the presence (upper panel) or absence (lower panel) of DTT. After incubation at $37^{\circ} \mathrm{C}$ for $20 \mathrm{~min}$, the proteins were separated by SDS-polyacrylamide gel electrophoresis, followed by immunoblotting with anti-Ubc9 antibody. (c) Inhibition of SUMOylation by compounds was counteracted by antioxidant $\mathrm{N}$-acetylL-cysteine (NAC) in vitro. Compounds at the indicated concentrations were incubated with the SUMOylation reaction mixture with or without 5 mM NAC, and then SUMOylated RanGAP1-C2 was detected as described in Figure 1b. 
we asked whether inhibition of protein SUMOylation by these quinone-related compounds is mediated by ROS generated from the quinone moieties in their structures. To explore this possibility, we first tested the effects of these compounds on disulfide formation between E1 and E2 because hydrogen peroxide generates a DTT-sensitive crosslink between E1 and E2 in the absence of ATP. ${ }^{12}$ For this purpose, wild-type or C173S mutant E1 and E2 proteins were incubated with or without the quinone compounds, or hydrogen peroxide in the absence of ATP, followed by immunoblotting with anti-Ubc9 antibody. In agreement with the previous report, ${ }^{12}$ DTT-sensitive high-MW species, probably attributable to the formation of a disulfide bond between E1 and E2, appeared following treatment with hydrogen peroxide (Figure 2b). It is noteworthy that these DTT-sensitive high-MW species were also detected in the presence of all quinone-containing compounds (Figure $2 b$ ), suggesting that these compounds induce a disulfide crosslink between E1 and E2. As the $\mathrm{C} 173 \mathrm{~S}$ mutant could also form the high-MW species, it seems possible that disulfide formation occurs independently of the catalytic cysteine residue. Finally, we examined effects of the antioxidant $N$-acetyl-L-cysteine (NAC) on the inhibition of in vitro SUMOylation by quinone-containing compounds. As shown in Figure 2c, all quinone-containing compounds inhibited SUMOylation of RanGAP1-C2 as expected, but their inhibitory effects were counteracted by NAC.

This study uncovered novel physiological activities of natural quinones, including nocardione $\mathrm{A}$ and its derivatives 3,3-DINORdunnione, 3,3-DINOR-dehydrodunnione and $\beta$-lapachone, revealing them as a novel class of SUMOylation inhibitors. Our results suggest the involvement of ROS, possibly generated by the quinone moiety, in the inhibition of SUMOylation by these compounds. Although the precise mechanism by which quinone-related compounds inhibit protein SUMOylation remains to be elucidated, our findings suggest that this inhibitory activity may be a general pharmacological activity of small-molecule compounds containing quinone in their structures. Consistent with this, kerriamycin B, an antibiotic reidentified as a SUMOylation inhibitor, also contains quinone in its structure. ${ }^{5}$

Both nocardione $\mathrm{A}$ and $\beta$-lapachone exhibit antiproliferative activities in cancer cells. ${ }^{13,14}$ Because recent studies implicated aberrant SUMO machinery in cancer development, the antitumor activity of quinone-containing compounds may be attributable, at least in part, to their ability to inhibit SUMO conjugation, in addition to the DNA damage induced through ROS formation. ${ }^{16}$ ARQ761, a clinical form of $\beta$-lapachone, is currently undergoing a clinical trial in patients with advanced solid tumors. Thus, these simple natural quinones are good starting materials for development of anti-cancer drugs.

\section{CONFLICT OF INTEREST}

The authors declare no conflict of interest.

\section{ACKNOWLEDGEMENTS}

This study was supported in part by the CREST Research Project, the Japan Science and Technology Corporation (JST), and the Japan Agency for Medical Research and Development (AMED) and by Grants-in-Aid for the Innovative Area 'Cancer'. This work was inspired by the Asian Chemical Biology Initiative.

1 Johnson, E. S. Protein modification by SUMO. Annu. Rev. Biochem. 73 355-382 (2004).

2 Eifler, K. \& Vertegaal, A. C. SUMOylation-mediated regulation of cell cycle progression and cancer. Trends. Biochem. Sci. 40, 779-793 (2015).

3 Krumova, P. \& Weishaupt, J. H. Sumoylation in neurodegenerative diseases. Cell Mol. Life Sci. 70, 2123-2138 (2013).

4 Fukuda, I. et al. Ginkgolic acid inhibits protein SUMOylation by blocking formation of the E1-SUMO intermediate. Chem. Biol. 16, 133-140 (2009).

5 Fukuda, I. et al. Kerriamycin B inhibits protein SUMOylation. J. Antibiot. 62 221-224 (2009).

6 Hirohama, M. et al. Spectomycin B1 as a novel SUMOylation inhibitor that directly binds to SUMO E2. ACS Chem. Biol. 8, 2635-2642 (2013).

7 Takemoto, M. et al. Inhibition of protein SUMOylation by davidiin, an ellagitannin from Davidia involucrata. J. Antibiot. 67, 335-338 (2014).

8 Kumar, A., Ito, A., Hirohama, M., Yoshida, M. \& Zhang, K. Y. Identification of quinazolinyloxy biaryl urea as a new class of SUMO activating enzyme 1 inhibitors. Bioorg. Med. Chem. Lett. 23, 5145-5149 (2013).

9 Kim, Y. S., Nagy, K., Keyser, S. \& Schneekloth, J. S. Jr. An electrophoretic mobility shift assay identifies a mechanistically unique inhibitor of protein sumoylation. Chem. Biol. 20, 604-613 (2013).

10 Kumar, A., Ito, A., Hirohama, M., Yoshida, M. \& Zhang, K. Y. Identification of sumoylation activating enzyme 1 inhibitors by structure-based virtual screening. J. Chem. Inf. Model. 53, 809-820 (2013).

11 Kumar, A., Ito, A., Hirohama, M., Yoshida, M. \& Zhang, K. Y. Identification of sumoylation inhibitors targeting a predicted pocket in Ubc9. J. Chem. Inf. Model. 54, 2784-2793 (2014).

12 Bossis, G. \& Melchior, F. Regulation of SUMOylation by reversible oxidation of SUMO conjugating enzymes. Mol. Cell. 21, 349-357 (2006).

13 Otani, T. et al. New Cdc25B tyrosine phosphatase inhibitors, nocardiones A and B, produced by Nocardia sp. TP-A0248: taxonomy, fermentation, isolation, structural elucidation and biological properties. J. Antibiot. 53, 337-344 (2000).

14 Schaffner-Sabba, K., Schmidt-Ruppin, K. H., Wehrli, W., Schuerch, A. R. \& Wasley, J. W. beta-Lapachone: synthesis of derivatives and activities in tumor models. J. Med. Chem. 27, 990-994 (1984).

15 Bolton, J. L., Trush, M. A., Penning, T. M., Dryhurst, G. \& Monks, T. J. Role of quinones in toxicology. Chem. Res. Toxicol. 13, 135-160 (2000).

16 Bey, E. A. et al. An NQO1- and PARP-1-mediated cell death pathway induced in nonsmall-cell lung cancer cells by beta-lapachone. Proc. Natl Acad. Sci. USA 104 11832-11837 (2007) 